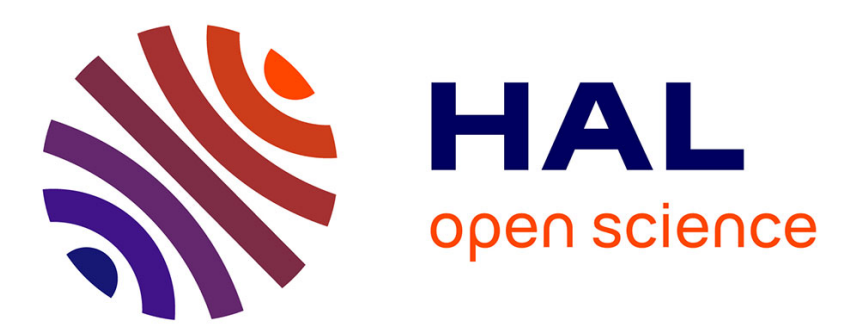

\title{
Effect of Fe doping on the terahertz conductivity of GaN single crystals
}

Filip Kadlec, Christelle Kadlec, Tanya Paskova, Keith Evans

\section{To cite this version:}

Filip Kadlec, Christelle Kadlec, Tanya Paskova, Keith Evans. Effect of Fe doping on the terahertz conductivity of GaN single crystals. Journal of Physics D: Applied Physics, 2010, 43 (14), pp.145401. 10.1088/0022-3727/43/14/145401 . hal-00597819

\section{HAL Id: hal-00597819 \\ https://hal.science/hal-00597819}

Submitted on 2 Jun 2011

HAL is a multi-disciplinary open access archive for the deposit and dissemination of scientific research documents, whether they are published or not. The documents may come from teaching and research institutions in France or abroad, or from public or private research centers.
L'archive ouverte pluridisciplinaire HAL, est destinée au dépôt et à la diffusion de documents scientifiques de niveau recherche, publiés ou non, émanant des établissements d'enseignement et de recherche français ou étrangers, des laboratoires publics ou privés. 


\title{
Effect of Fe doping on the terahertz conductivity of GaN single crystals
}

\author{
Filip Kadlec, Christelle Kadlec \\ Institute of Physics, Academy of Sciences of the Czech Republic, Na Slovance 2, \\ 18221 Prague 8, Czech Republic
}

\section{Tanya Paskova, Keith Evans}

Kyma Technologies, Inc., Raleigh, NC 27617, USA

\begin{abstract}
Bulk single crystals of GaN with different degree of Fe doping were studied using time-domain terahertz spectroscopy at high temperatures. Features due to free carriers were observed in the complex permittivity spectra with a pronounced dependence on both doping and temperature. Fitting the spectra using the Drude model made it possible to deduce the defect ionization energy of $16 \mathrm{meV}$ in the undoped sample while the spectra of doped samples are consistent with an ionization energy of $60 \mathrm{meV}$. Also, the free carrier concentrations at temperatures from $300 \mathrm{~K}$ to $900 \mathrm{~K}$ were estimated.
\end{abstract}

Keywords: Gallium nitride, terahertz spectroscopy, iron doping, self-heating PACS numbers: $78.30 . F s$

Submitted to: J. Phys. D: Appl. Phys. 


\section{Introduction}

Gallium nitride is a technologically important material with a wide range of both present and prospective applications. Since the success in epitaxial growth of GaN thin films (see Ref. [1] for a review), it has been largely employed in light-emitting diodes, LED displays, and lasers. In the last years, there has been a rapid development in technologies aiming at the production of bulk GaN; its future uses include e.g. high-power high-frequency operating field effect transistors, radars and power amplifiers [2]. The epitaxial growth often uses sapphire substrates, and the technology leads to an unintentional $n$-doping of $\mathrm{GaN}$; $\mathrm{O}$ and $\mathrm{Si}$ are the main residual impurities at the origin of de conductivity [3]. For compensation of these donors, doping by Fe can be used; Fe acceptors substitute for Ga and substantially increase the resistivity [4]. In order to achieve optimal properties of the doped crystals, a deeper knowledge of the underlying physical properties of bulk GaN, namely their conductivity mechanisms, is required. From the point of view of high-power applications which may lead to self-heating [5], the properties at higher temperatures are of particular importance. However, standard transport measurements are challenging owing to difficulties in producing low resistivity contacts to the highlyresistive GaN material.

Terahertz $(\mathrm{THz})$ spectroscopy is an alternative technique and a convenient tool for studying the dynamical conductivity of GaN. It was shown that the free carriers follow the Drude model [6] with a plasma frequency in the $\mathrm{THz}$ range. Small deviations from the Drude model were reported and studied [7], and the $\mathrm{THz}$ spectra were shown to depend on the carrier concentration [8]. In the present paper, we study experimentally the $\mathrm{THz}$ response of bulk GaN crystals with varying Fe doping and at variable temperatures.

\section{Sample preparation}

The samples were grown in a vertical hydride vapor phase epitaxy hot-wall reactor at a sub-atmospheric pressure. The single crystals were deposited at a rate of $150 \mu \mathrm{m} / \mathrm{h}$ on $c^{-}$ plane (0001) sapphire substrates with an AlN template layer, in the downstream part of the reactor where $\mathrm{GaCl}$ and $\mathrm{NH}_{3}$, transported by the $\mathrm{N}_{2}$ gas react. The substrates were maintained at a temperature of $T \approx 1000^{\circ} \mathrm{C}$. As a Fe source for doping, a variable flow of bis(cyclopentadienyl)iron was introduced into the reactor via $\mathrm{N}_{2}$ carrier gas. Once the substrates removed, the GaN crystals were cut and polished to the shape of slabs with optical quality surfaces and thicknesses ranging from 0.5 to $1.2 \mathrm{~mm}$. Four different samples were measured: one undoped, No. 1 (AH939), and three doped, Nos. 2-4 (AE492, AE535 and AE782; these fabrication labels were used also in refs. [9, 10]). Their Fe atom concentrations were determined by means of secondary ion mass spectrometry as $3 \times 10^{17}, 7 \times 10^{17}$ and $3 \times 10^{18} \mathrm{~cm}^{-3}$, respectively. 


\section{Experimental details}

Our custom-made experimental setup for time-domain $\mathrm{THz}$ spectroscopy was based on a Ti:sapphire laser oscillator providing $810 \mathrm{~nm}, 80 \mathrm{fs}$ pulses at a $76 \mathrm{MHz}$ repetition rate. The pulses excited an interdigitated photoconducting emitter [11] which generated linearly polarized $\mathrm{THz}$ pulses. A weak part of the laser beam was directed on a $1 \mathrm{~mm}$ thick $\langle 110\rangle$ cut ZnTe crystal for electro-optic sampling of the transmitted $\mathrm{THz}$ waves. The whole $\mathrm{THz}$ beam was enclosed in a vacuum chamber (about $20 \mathrm{mbar}$ ) in order to avoid water vapor absorption. The samples were placed in a furnace with optical ports, using a clear aperture of 3 to $7 \mathrm{~mm}$, and heated up to $T=900 \mathrm{~K}$ with steps of $100 \mathrm{~K}$, except for the sample No. 1 where the temperature of $400 \mathrm{~K}$ was replaced by 360 and $430 \mathrm{~K}$. The instrument covered a frequency range of $0.25-2.5 \mathrm{THz}$.

\section{Results and evaluation}

From the complex transmittance, we calculated numerically the spectra of complex refractive index $\hat{N}(\nu)$ which we converted to permittivity $\hat{\varepsilon}(\nu) \equiv \varepsilon^{\prime}+\mathrm{i} \varepsilon^{\prime \prime}=\hat{N}^{2}(\nu)$ and conductivity spectra, $\hat{\sigma}(\nu)=-2 \pi \nu \mathrm{i} \varepsilon_{0} \hat{\varepsilon}(\nu) ; \varepsilon_{0}$ denotes the vacuum permittivity. Symbols in Fig. 1 show the real parts, $\varepsilon^{\prime}(\nu)$ and $\sigma^{\prime}(\nu)$. Signatures of free carrier transport can be clearly seen - even a relatively weak conductivity shows up as a non-vanishing $\sigma^{\prime}$ in the low-frequency part. This feature is the strongest for the undoped sample where it clearly gives rise to a Drude peak and it and decreases upon Fe doping. For the highest doped sample (No. 4), no signs of free charge carriers are visible at any temperature.

As a common trend, the $\varepsilon^{\prime}(\nu)$ spectra display a monotonic increase upon heating. For all Fe doped samples, they are almost flat, except for small experimental artifacts and/or noise. In the upper frequency parts of the spectra of samples (No. 3, 47) with higher doping (Fig. 1e, g), an increase with frequency is seen, similar to previous observations [8]. We attribute this to a contribution of a broad phonon absorption beyond our accessible frequency range.

This term also influences the $\sigma^{\prime}(\nu)$ (i.e., $\varepsilon^{\prime \prime}(\nu)$ ) spectra in their high-frequency part. From the fitting described below it appears clearly that without such a term, the conductivity increase above $\nu>1 \mathrm{THz}$ would not be observed.

The most interesting information, however, is related to the low-frequency parts of $\sigma^{\prime}(\nu)$; it is that about free charge carriers. The temperature dependence of these low-frequency parts is strongly influenced by doping. While it decreases with $T$ in the sample No. 1 (Fig. 1b), an increase upon heating is observed in the lower Fe doped samples No. 2 and No. 3 (Fig. 1d, f); in the latter case, heating up is necessary to bring up the Drude-like contribution. Finally, for the sample No. 4, the free carrier conductivity is not visible at any temperature (Fig. 1h).

We fitted the $\hat{\varepsilon}(\nu)$ spectra of the samples displaying signs of electrical conduction. In view of the observed features, we found the same model as used in Ref. [8] to be the 


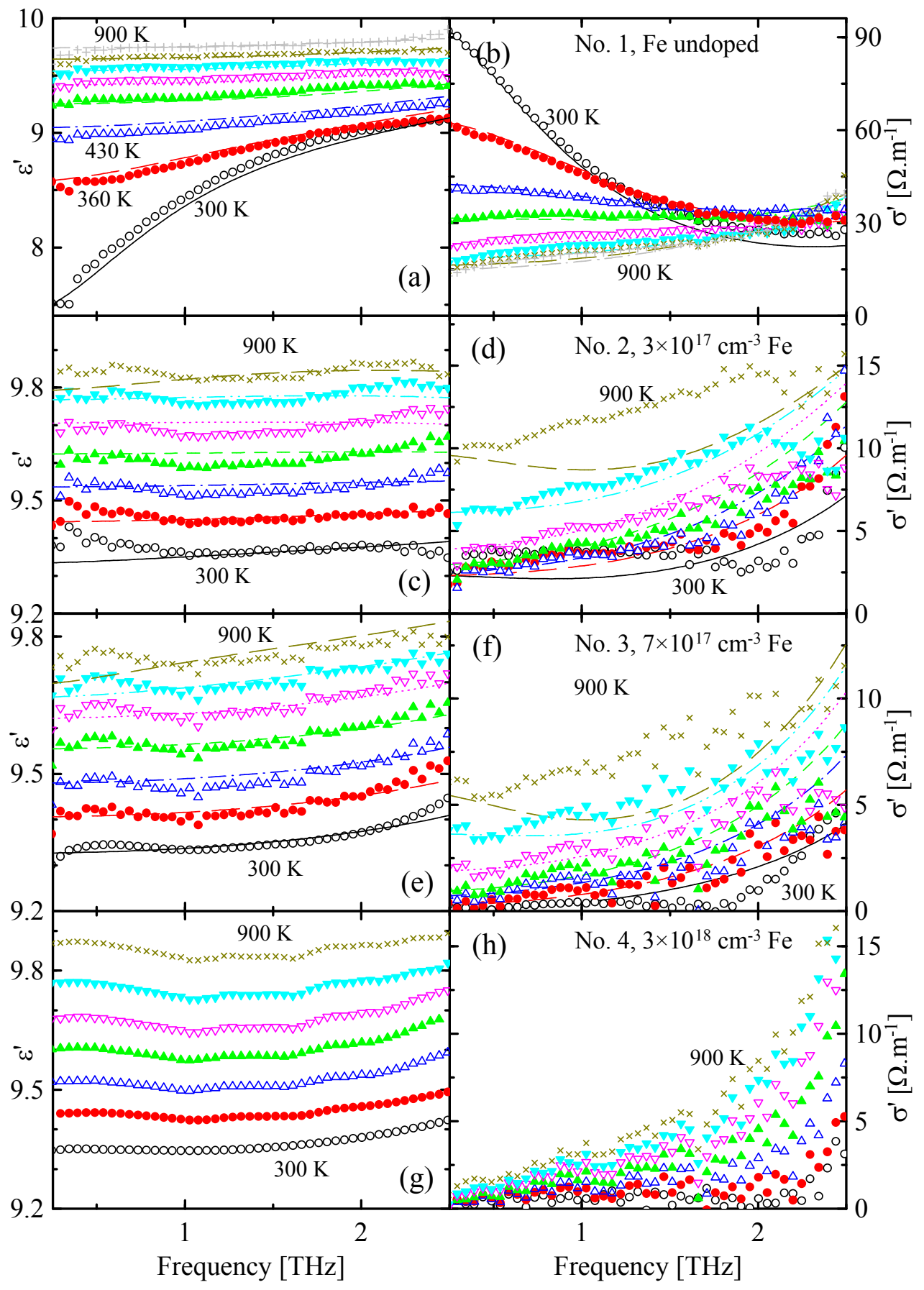

Figure 1. Real permittivity (left panels) and conductivity (right panels) spectra of the samples. Points represent experimental data and lines show fits by using Eq. 1. 


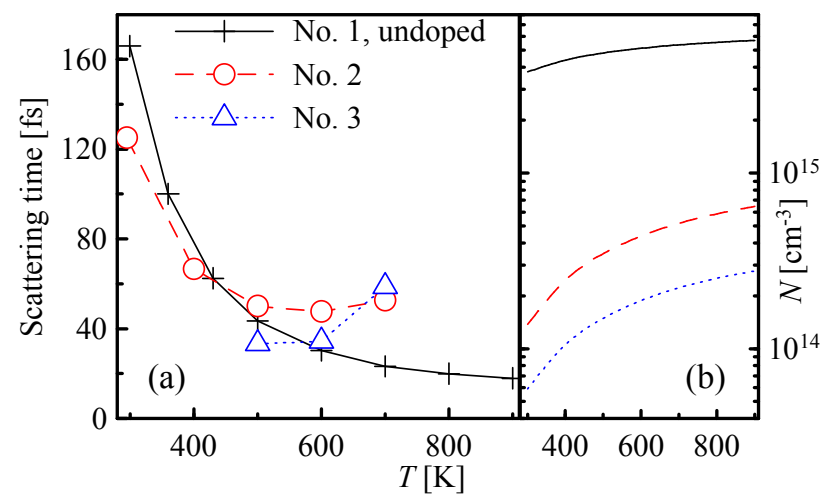

Figure 2. Free carrier scattering times $\tau$ (a) and concentrations (b) estimated from spectra fitting as a function of $T$.

best suited $(\omega=2 \pi \nu)$ :

$$
\hat{\varepsilon}(\omega)=\frac{\omega_{\mathrm{p}}^{2}}{\omega^{2}-\mathrm{i} \omega \gamma}+\frac{f_{\mathrm{o}}}{\omega_{0}^{2}-\omega^{2}-\mathrm{i} \omega \Gamma}+\varepsilon_{\infty}
$$

with the plasma frequency given by $\omega_{\mathrm{p}}^{2}=N e_{0}^{2} / m_{0} m^{*} \varepsilon_{0}$. Here, $e_{0}$ and $m_{0}$ denote the free electron charge and mass, respectively. Eq. 1 contains two frequency-dependent terms: (i) a Drude-like plasma of free charge carriers with a concentration $N$, effective mass $m^{*}$ and a mean collision rate $\gamma$ and (ii) an overdamped harmonic oscillator with oscillator strength $f_{0}$, eigenfrequency $\omega_{0}$ and damping $\Gamma$, describing the broad features at higher frequencies. The constant $\varepsilon_{\infty}$ accounts for contributions of all modes far beyond the $\mathrm{THz}$ region.

First, the complex room-temperature spectrum of the undoped sample (No. 1) alone was fitted; the result is shown by solid lines in Fig. 1a, b. The extrapolated value of static conductivity is $\sigma_{\mathrm{DC}} \approx 1(\Omega \cdot \mathrm{cm})^{-1}$. Owing to the pronounced slope in the real part, it is possible to reliably determine the angular plasma frequency $\omega_{\mathrm{p}}=8.3 \times 10^{12} \mathrm{rad} \mathrm{s}^{-1}$ and the scattering time $\tau \equiv 1 / \gamma=160$ fs. Assuming that the free charge carriers are electrons with an effective mass [12] of $m^{*}=0.2$, one can calculate their concentration, $N(300 \mathrm{~K})=4 \times 10^{15} \mathrm{~cm}^{3}$ and mobility $\mu=1440 \mathrm{~cm}^{2} / \mathrm{V} \cdot \mathrm{s}$. These results are in agreement with values determined by $\mathrm{THz}$ spectroscopy and reported earlier for undoped GaN [6].

Further, we performed a common fit of the spectra at all temperatures. We assumed a linear temperature dependence of $\varepsilon_{\infty}$ and the oscillator tranverse frequency was fixed to $\omega_{0} / 2 \pi=5 \mathrm{THz}$; its strength was assumed to be constant in $T$ and its damping $\Gamma$ linearly proportional to $T$. Thus, it was possible to describe the high-frequency part of the spectra. In the weak-doping approximation, the concentration of free electrons can be written as $N(T)=N_{0} \exp \left(-E_{\mathrm{D}} / k_{\mathrm{B}} T\right)$ where $E_{\mathrm{D}}$ is the defect ionization energy and $k_{\mathrm{B}}$ the Boltzmann constant. The collision rate $\gamma$ was allowed to vary with $T$ without limitations. The solid lines in Fig. 1a, b show the fit matching well the experimental data; the best match is found for a value of $E_{\mathrm{D}}=16 \pm 3 \mathrm{meV}$. Solid lines in Fig. 2 show the obtained functions $\tau(T)$ and $N(T)$.

The spectra of the sample No. 2 with low Fe doping display much smaller 
spectral features than those of the undoped sample (No.1). Thus, it was necessary to fit them together; we used the same model (Eq. 1) and temperature dependence of parameters. We found the room-temperature concentration of electrons to be $N(300 \mathrm{~K})=1.4 \times 10^{14} \mathrm{~cm}^{3}$, while the defect ionization energy is substantially higher, $E_{\mathrm{D}}=60 \pm 10 \mathrm{meV}$. This leads to a steeper increase of $N$ upon heating (Fig. 2b). The model does not agree very well with the experimental data at the highest temperatures $(T>700 \mathrm{~K})$, which is probably a sign of a supplementary absorption mechanism in this temperature range. Therefore, the values of the scattering time $\tau$ become unreliable and they are not shown in Fig. 2a. Nevertheless, since the model describes well the conductivity spectra in the lowest part of the $\mathrm{THz}$ range, it should still provide a useful estimate of the free carrier concentration up to the highest temperatures.

In the sample No. 3, the even higher level of doping suppresses features linked to the free carrier absorption at room-temperature (Fig. 1e, f). However, upon heating above $500 \mathrm{~K}, \sigma^{\prime}$ increases at lower frequencies. Due to its low values, the fit performed for this sample does not provide unambiguously the concentration of the free electrons. Therefore, we assumed a concentration inversely proportional to the Fe doping level, i.e. $N(300 \mathrm{~K})=6 \times 10^{13} \mathrm{~cm}^{3}$. In this way, with the same value of $E_{\mathrm{D}}=60 \mathrm{meV}$, a good agreement with the measured spectra was achieved. Analogously to the sample No. 2, the validity of the model at the highest temperatures is limited; thus, values of $\tau$ can be estimated only in the range $500 \mathrm{~K} \leq T \leq 700 \mathrm{~K}$.

Finally, no features related to electrical conductivity are observed in the spectra of the sample No. 4 with the highest doping. By comparing the spectra at highest temperatures with those of the sample No. 3, and assuming the same values of $\gamma$, we can estimate that the carrier concentration decreased by a factor of four or more.

\section{Discussion}

In the undoped sample, the activation energy of $16 \mathrm{meV}$ is very close to that determined from transport measurements in intentionally Si-doped samples [13]. By contrast, the $\mathrm{THz}$ mobility is one order of magnitude higher, while the room-temperature concentration of carriers in $\mathrm{THz}$ experiments is about two orders of magnitude lower. This means that the interactions of carriers dominantly contributing to the $\mathrm{THz}$ conductivity, where local motion across about tens of nanometers is sensed, are different from those occurring in the long-range transport; we attribute this fact to short-scale spatial doping inhomogeneities. The energy level of $16 \mathrm{meV}$ apparently corresponds to the residual donor-either $\mathrm{O}$ or $\mathrm{Si}$ - as reported earlier based on Hall-mobility measurements on electron-irradiated samples [14]. The activation energy of $60 \mathrm{meV}$ in doped samples (Nos. 2 and 3) is clearly linked to the Fe doping; in agreement with previous works $[14,15]$ we attribute it to the $\mathrm{N}$ vacancy donor $\mathrm{V}_{\mathrm{N}}$. The concentration of these donors appears to increase at low level of Fe doping; this might be because the doping leads to a decrease of the Fermi level whereby the vacancies would become energetically more stable. In this way, Fe atoms could stabilize the $\mathrm{N}$ vacancies, or 
even $\mathrm{Fe}-\mathrm{V}_{\mathrm{N}}$ complexes might form. Since the Fig. 3 of Ref. [4] displays only a partial agreement between experimental and simulated EXAFS spectra, a part of the Fe dopants probably occupy other crystal sites. Therefore, our hypotheses are not in contradiction with the observation of $\mathrm{Fe}_{\mathrm{Ga}}$ reported in Ref. [4].

For the undoped sample (No. 1), $\tau(T)$ data obtained from fits (Fig. 2) show a monotonic decrease, apparently linked to the electron-phonon scattering increasing on heating. For the low doped sample (No. 2), the same trend is observed at lower temperatures, however, around $600 \mathrm{~K}, \tau$ seems to level off. Upon heating above $700 \mathrm{~K}$, the model described by Eq. 1 departs from our experimental data, therefore $\tau$ cannot be reliably determined. This holds also for the higher doped sample (No. 3); values of $\tau$ similar to those in samples No. 1, 2 are observed at medium temperatures. For sample No. 3 at low temperatures, $\tau$ cannot be determined owing to the absence of the Drude contribution. The deviation of our model from the data at the highest temperatures suggests that the energy scheme may be more complex than that we take into account; however, the current knowledge of the energy levels in Fe:GaN and the accuracy of our measurements do not enable us to set up a more appropriate model.

We also note that, as $\sigma^{\prime}(\nu)$ according to the Drude model rises steeply towards low frequencies, measurements at microwave frequencies might provide even more sensitivity for doped samples.

\section{Conclusion}

In conclusion, we have studied the impact of Fe doping on the $\mathrm{THz}$ spectra of GaN crystals in view of their future use above room temperature. While the doping leads to a suppression of conductivity at room temperature, heating the crystals has the opposite effect. From the temperature dependences of the spectra, we have determined defect ionization energies consistent with those observed earlier and temperature dependences of the free carrier concentrations. Our results are expected to contribute to both the fundamental understanding of carrier transport and the technological use of GaN crystals.

\section{Acknowledgment}

Support by the Czech Academy of Sciences (project AVOZ 10100520) as well as by MDA under the HQ0147-09-C-0005 contract for bulk GaN characterization is acknowledged. We would like to thank Dr. David Look for useful discussions.

\section{References}

[1] S. J. Pearton, C. R. Abernathy, and Fan Ren. Gallium nitride processing for electronics, sensors and spintronics. Springer, 2006. 
[2] S. Keller, Yi-Feng Wu, G. Parish, Naiqian Ziang, J.J. Xu, B.P. Keller, S.P. DenBaars, and U. K. Mishra. Gallium nitride based high power heterojunction field effect transistors: Process development and present status at ucsb. IEEE Trans. on Electron Devices, 48(3):552-558, 2001.

[3] W. J. Moore, Jr. J. A. Freitas, G. C. B. Braga, R. J. Molnar, S. K. Lee, K. Y. Lee, and I. J. Song. Identification of $\mathrm{Si}$ and $\mathrm{O}$ donors in hydride-vapor-phase epitaxial GaN. Applied Physics Letters, 79(16):2570-2572, 2001.

[4] Yoshinao Kumagai, Fumitaka Satoh, Rie Togashi, Hisashi Murakami, Kikurou Takemoto, Junji Iihara, Koji Yamaguchi, and Akinori Koukitu. Fe-doped semi-insulating GaN substrates prepared by hydride vapor-phase epitaxy using GaAs starting substrates. Journal of Crystal Growth, 296(1):11 - 14, 2006.

[5] M. Asif Khan, J. W. Yang, W. Knap, E. Frayssinet, X. Hu, G. Simin, P. Prystawko, M. Leszczynski, I. Grzegory, S. Porowski, R. Gaska, M. S. Shur, B. Beaumont, M. Teisseire, and G. Neu. GaNAlGaN heterostructure field-effect transistors over bulk GaN substrates. Applied Physics Letters, $76(25): 3807-3809,2000$.

[6] W. Zhang, Abul K. Azad, and D. Grischkowsky. Terahertz studies of carrier dynamics and dielectric response of n-type, freestanding epitaxial GaN. Applied Physics Letters, 82(17):2841$2843,2003$.

[7] Tsong-Ru Tsai, Shi-Jie Chen, Chih-Fu Chang, Sheng-Hsien Hsu, Tai-Yuan Lin, and Cheng-Chung Chi. Terahertz response of GaN thin films. Opt. Express, 14(11):4898-4907, 2006.

[8] H. C. Guo, X. H. Zhang, W. Liu, A. M. Yong, and S. H. Tang. Terahertz carrier dynamics and dielectric properties of GaN epilayers with different carrier concentrations. Journal of Applied Physics, 106(6):063104, 2009.

[9] Z.-Q. Fang, B. Claflin, D. C. Look, S. Elhamri, H. E. Smith, W. C. Mitchel, D. Hanser, E. A. Preble, and K. R. Evans. Deep centers in semi-insulating Fe-doped native GaN substrates grown by hydride vapour phase epitaxy. physica status solidi (c), 5:1508-1511, 2008.

[10] J.A. Freitas Jr., M. Gowda, J.G. Tischler, J.-H. Kim, L. Liu, and D. Hanser. Semi-insulating gan substrates for high-frequency device fabrication. Journal of Crystal Growth, 310(17):3968 $-3972,2008$.

[11] A. Dreyhaupt, S. Winnerl, T. Dekorsy, and M. Helm. High-intensity terahertz radiation from a microstructured large-area photoconductor. Applied Physics Letters, 86(12):121114, 2005.

[12] I. Vurgaftman and J. R. Meyer. Band parameters for nitrogen-containing semiconductors. Journal of Applied Physics, 94(6):3675-3696, 2003.

[13] H. M. Ng, D. Doppalapudi, T. D. Moustakas, N. G. Weimann, and L. F. Eastman. The role of dislocation scattering in n-type GaN films. Applied Physics Letters, 73(6):821-823, 1998.

[14] D. C. Look, D. C. Reynolds, J. W. Hemsky, J. R. Sizelove, R. L. Jones, and R. J. Molnar. Defect donor and acceptor in GaN. Phys. Rev. Lett., 79(12):2273-2276, Sep 1997.

[15] D. C. Look, G. C. Farlow, P. J. Drevinsky, D. F. Bliss, and J. R. Sizelove. On the nitrogen vacancy in GaN. Applied Physics Letters, 83(17):3525-3527, 2003. 